\title{
Analysis and Evaluation of the Dynamic Performance of SMA Actuators for Prosthetic Hand Design
}

\author{
Kevin O'Toole \\ Technological University Dublin, kevin.otoole@tudublin.ie \\ Mark McGrath \\ Technological University Dublin, mark.mcgrath@tudublin.ie \\ Eugene Coyle \\ Technological University Dublin, Eugene.Coyle@tudublin.ie
}

Follow this and additional works at: https://arrow.tudublin.ie/engschmanart

Part of the Mechanical Engineering Commons

\section{Recommended Citation}

O'Toole, K., McGrath, M., Coyle, E.: Analysis and evaluation of the dynamic performance of SMA actuators for prosthetic hand design. Journal of Materials Engineering and Performance, Vol. 18, issues 5-6, pp.

781-786. 2009. doi:10.21427/amsq-vx78

This Article is brought to you for free and open access by the School of Manufacturing and Design Engineering at ARROW@TU Dublin. It has been accepted for inclusion in Articles by an authorized administrator of ARROW@TU Dublin. For more information, please contact arrow.admin@tudublin.ie, aisling.coyne@tudublin.ie, gerard.connolly@tudublin.ie.

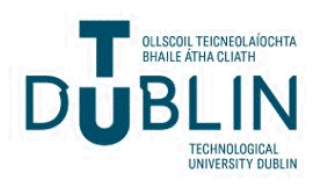




\title{
Analysis \& Evaluation of the Dynamic Performance of SMA Actuators for Prosthetic Hand Design
}

Kevin T. O’Toole, Mark M. McGrath, Eugene Coyle

\begin{abstract}
It is widely acknowledged within the biomedical engineering community that Shape Memory Alloys (SMA's) exhibit great potential for application in the actuation of upper limb prosthesis designs. These lightweight actuators are particularly suitable for prosthetic hand solutions. A four-fingered, twelve degree-of-freedom prosthetic hand has been developed featuring SMA bundle actuators embedded within the palmar structure. Joule-heating of the SMA bundle actuators generates sufficient torque at the fingers to allow a wide range of everyday tasks to be carried out. Transient characterisation of SMA bundles has shown that performance/response during heating and cooling differs substantially. Natural convection is insufficient to provide for adequate cooling during elongation of the actuators.

An experimental test-bed has been developed to facilitate analysis of the heat transfer characteristics of the appropriately sized SMA bundle actuators for use within the prosthetic hand design. Various modes of heat-sinking are evaluated so that the most effective wire-cooling solution can be ascertained. SMA bundles of varying size will be used so that a generalised model of the SMA displacement performance under natural and forced cooling conditions can be obtained. The optimum cooling solution will be implemented onto the mechanical hand framework in future work. These results, coupled with phenomenological models of SMA behaviour, will be used in the development of an effective control strategy for this application in future work.
\end{abstract}

\section{Nomenclature}

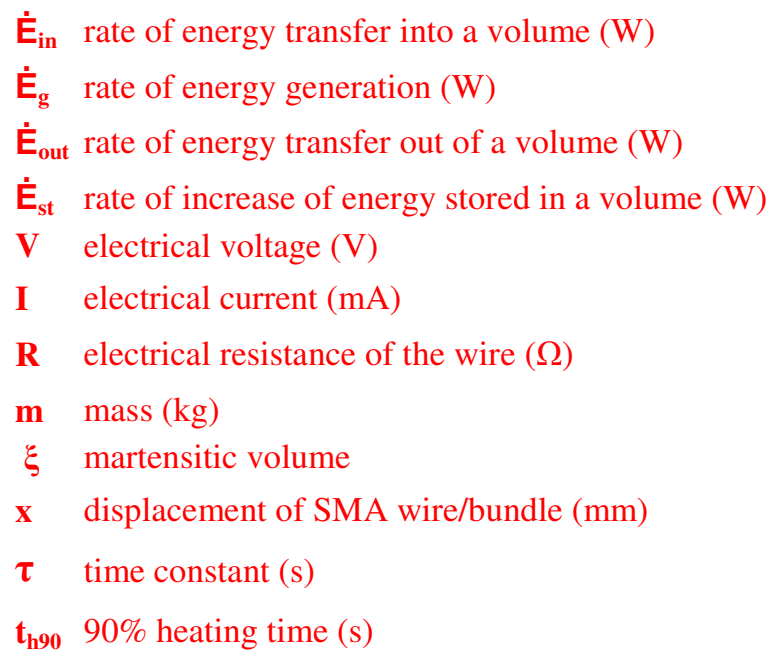

T temperature $\left({ }^{\circ} \mathrm{C}\right)$

$\Delta \mathbf{T}$ temperature difference $\left(\mathrm{T}_{\mathrm{w}}-\mathrm{T}_{\infty}\right)\left({ }^{\circ} \mathrm{C}\right)$

A surface area of SMA wire $\left(\mathrm{m}^{2}\right)$

h heat transfer coefficient $\left(\mathrm{W} / \mathrm{m}^{2} . \mathrm{K}\right)$

k thermal conductivity (W/m.K)

H latent heat $(\mathrm{kJ} / \mathrm{kg})$

$\mathbf{T}_{\mathbf{w}}$ wire surface temperature $\left({ }^{\circ} \mathrm{C}\right)$

$\mathbf{T}_{\infty}$ ambient temperature $\left({ }^{\circ} \mathrm{C}\right)$

f frictional force $(\mathrm{N})$

$\mathbf{c}_{\mathbf{p}}$ specific heat at constant pressure (J/kg.K)

$\mathbf{t}_{\mathbf{c 9 0}}$ 90\% cooling time (s)

P electrical power (W) 


\section{Introduction}

Shape memory alloys (SMAs) have the potential to revolutionise the field of biomedical engineering, in particular, lower arm prosthesis design. Currently, most lower arm prostheses are driven by electromechanical DC motors [1-5]. DC motors inhibit aspects of prosthesis design owing to their size and weight. As a result, traditional intelligent prosthetics tend to be bulky, heavy and noisy. Consequently, designers have been focusing their attention on various alternative actuation techniques. SMAs exhibit favourable properties such as low weight, quiet operation, low cost and high force to weight ratios. However, SMAs exhibit a significant hysteresis during the heating and cooling cycles which can pose problems in design, operation and control.
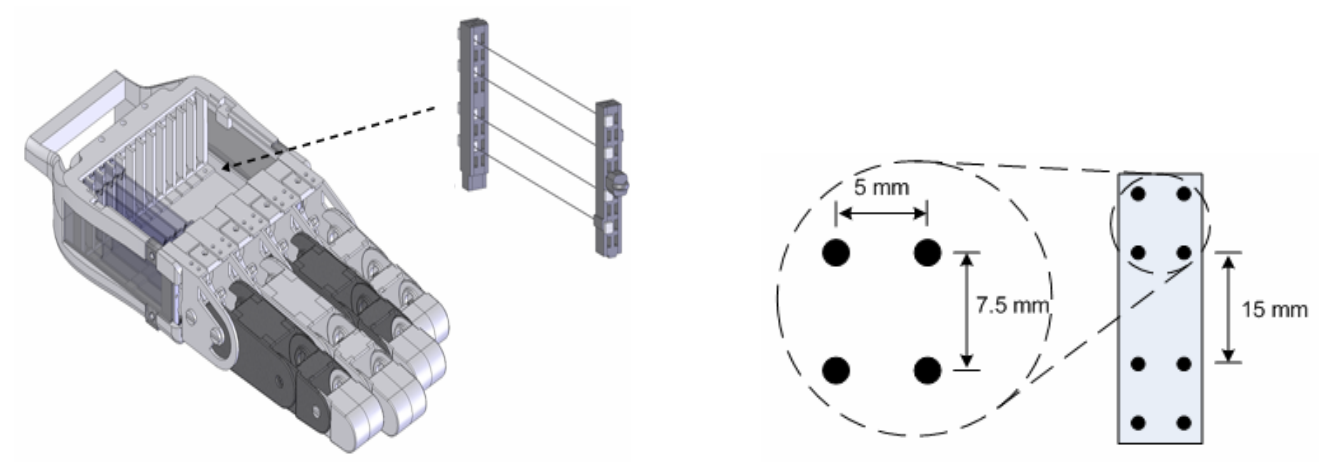

Figure 1: SMA Actuated Hand Prototype \& SMA Bundle Figure 2: SMA Bundle Dimensions

A four fingered hand prosthesis featuring embedded SMA bundle actuators has been designed by this group (Figure 1) [6] requiring $0.66 \mathrm{~W} / 150 \mu \mathrm{m}$ SMA for maximum safe contraction rate. Within the prosthetic design, the bundle actuators are appropriately sized to give the force requirement at the various phalanges of the human hand. The goal of this work is to optimise the transient performance of the SMA actuators so that they can deliver a response comparable to that of a human muscle performing everyday tasks. Six critical steps for full characterisation of SMA wire bundles for use in prosthesis design are outlined in Table 1. This paper focuses on steps 2-4, which examine the relationship between heat transfer and strain response in SMAs. These characteristics are intrinsically linked, and by maximising the heat transfer to and from the SMA, the strain response can be optimised. This is further illustrated in Section 4 of this work where the strain response is directly compared with the thermal response. 
Table 1: SMA Characterisation: 6 Stages

\begin{tabular}{|c|l|c|}
\hline 1 & Strain characterisation of SMA wire \& SMA bundle actuator & Completed in previous work [7] \\
\hline 2 & Heat transfer analysis of SMA wire \& SMA bundle actuator & Current work \\
\hline 3 & Analysis of SMA performance during heating/cooling cycle & Current work \\
\hline 4 & Evaluation of forced cooling methods & Current work \\
\hline 5 & Dynamic response of SMA bundle actuator during cyclic testing & Preliminary work completed \\
\hline 6 & Fatigue testing of SMA bundle actuators & Future Work \\
\hline
\end{tabular}

This paper is laid out as follows: Section 2 describes the design and manufacture of the test rig employed in this work. Section 3 analyses the heat transfer to/from single SMA wires and SMA bundle actuators. Section 4 explores the complex relationship between SMA wire temperature, strain response and force generation in SMA bundle actuators. Section 5 outlines the testing of a suitable forced cooling technique with a view to minimising the hysteresis associated with SMAs. Some conclusions as well as future work direction are outlined in Section 6.

\section{Test Bed Development}

A test rig was developed to facilitate testing of both single SMA wires and various SMA bundle sizes (Figure 3). A cooling system module, developed as a modular attachment to the rig, provides for forced cooling (Figure 7). The rig can be used in an open or enclosed state depending upon the nature and objectives of each individual experiment. Bundles of different sizes, and springs of different stiffness (to provide the relaxation force required) can be inserted to cater for a range of experimental testing.

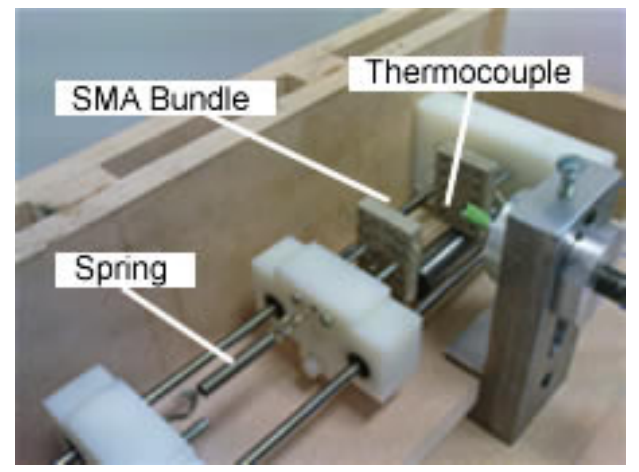

Figure 3: SMA Layout in Test Rig (h-value setup)

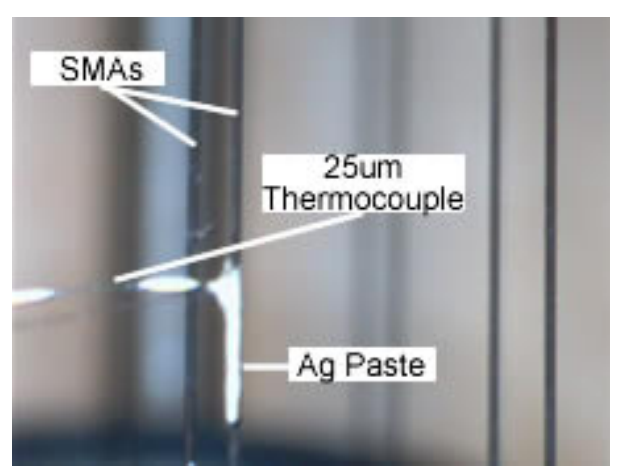

Figure 4: Sensory System Layout

Various sensors are used in the real-time detection of the changing physical phenomena during the experiments. Two K-type micro-thermocouples $(25 \mu \mathrm{m})$ are used to measure wire temperature and 
ambient air temperature respectively (Figure 4). A Solartron LVDT is employed in the measurement of the rate of contraction and expansion of the SMA wire/bundle under thermal excitation. External noise is filtered using a $4^{\text {th }}$ order Butterworth filter. Current and voltage readings are also recorded. A NI SCC-68 DAQ is used to condition the outputs from each individual sensor. The results are displayed in NI LabVIEW and compiled in Matlab.

\section{SMA Heat Transfer}

\subsection{Conservation of Energy \& Heat Transfer Analysis}

The heating and cooling cycles of SMA actuators follow the law of conservation of energy for a control volume. This law is defined as:

$\dot{E}_{\text {in }}+\dot{E}_{g}+\dot{E}_{\text {out }}=\dot{E}_{\text {st }}$

Szykowny et al stated that the energy dissipation takes place mostly in the form of heat convection [8]. Previous groups $[9,10]$ have shown that the SMA wire heat transfer model can be stated as:

$$
V I-h(T) A\left[T_{w}-T_{\infty}\right]-m \Delta H \frac{d \xi}{d t}-\mathrm{f} \frac{d x}{d t}=m C_{p} \frac{d T}{d t}
$$

An additional term has been added to the LHS of (2) to account for the power required to overcome the damping effect of the test rig during transient SMA straining activity. By comparing (1) with (2) it can be concluded that during the transient stage of the SMA contraction:

$$
\begin{aligned}
& \dot{E}_{\text {in }}=V I \\
& \dot{E}_{g}=0 \\
& \dot{E}_{\text {out }}=h(T) A\left[T_{w}-T_{\infty}\right]+m \Delta H \frac{d \xi}{d t}+\mathrm{f} \frac{d x}{d t} \\
& \dot{E}_{s t}=m C_{p} \frac{d T}{d t}
\end{aligned}
$$

When the steady-state stage of the cycle is reached, the heat transfer equation can be reduced to

$$
\dot{E}_{\text {in }}=V I=h A\left[T_{w}-T_{\infty}\right]
$$

The transient performance must be optimised in order to maximise the dynamic performance of the SMA bundle actuator. Previous work by this group has shown that the heating and cooling time responses of SMA bundles differ significantly [7]. Measures must be taken to move towards 
comparable performance during each stage of the cycle. This can be accomplished by improving the rate of cooling (Section 5).

The heat transfer coefficient, $h$, is an important consideration when completing the characterisation of single SMA wires and SMA wire bundles. This is particularly important in prosthesis design as the SMA bundles are located in an enclosed environment (within the hand). The additional heat convected into this environment by the SMA bundles must be accounted for and removed so that adequate control of actuation can be achieved. Szykowny et al have suggested that the value of $h$ for a single SMA wire decreases with increasing temperature in an open environment [8]. They have also demonstrated that the calculation of $h$ for a single SMA wire using established analytical methods will yield large error margins suggesting that these methods are unsuitable. The current work aims to establish a correlation between $h$ for a single SMA wire and a bundle of SMA wires placed in a parallel arrangement (Figures $2 \& 3$ ).

\subsection{Determination of Heat Transfer Coefficient for a SMA bundle}

The initial testing focused on establishing $h$ for a single SMA wire. This was carried out with the test rig fully sealed to maintain a steady ambient temperature of approximately $22^{\circ} \mathrm{C}$. The wire surface temperature and ambient temperature were continually recorded. Upon completion, the 8-wire SMA bundle (Figure 3) was loaded into the rig and testing was repeated. Wire surface temperature was taken at one point on the SMA wire located at the top left of the bundle. Ambient temperature was recorded 200mm from the SMA bundle, which is well outside the expected thermal boundary region of the SMA bundle. The $h$ value can then be determined from eqn (8).

$$
h=\frac{V I}{A\left[T_{w}-T_{\infty}\right]}
$$

\subsection{Results}

The $h$ value was found to increase slightly in a linear fashion as input power is increased. The $h$ values established for the single wire and wire bundle are similar in the SMA active region (200mA - 400mA) with values in the range of $200-250 \mathrm{~W} / \mathrm{m}^{2} . \mathrm{K}$, and followed the linear relationship shown in (9).

$h=11.6 P+180$ 
The results indicate that the thermal boundary regions of each individual SMA wire within the bundle do not interact substantially with the bundle dimensions used in this work. The force generated remains 8 times that of a single SMA wire. The results demonstrate that the thermal analysis of SMA bundles can be carried out by considering the parallel wires to be lined up in series. This approach can only be used if the physical distance between adjacent wires is greater than the thermal boundary layer thickness for each SMA wire in the bundle.

\section{Relationship between Thermal Response \& Strain Response}

\section{a) Fixed Bundle Size - Varying Current}

It is important to note that as the SMAs undergo transformation from the martensitic state to the austenitic state, the electrical resistance changes due to the differing properties of martensite and austenite. This change is relatively small and as a result, the electrical resistance of an SMA bundle with a fixed number of wires can be considered constant during the following analysis. Experimental results suggest that

$$
\frac{d(\Delta T)}{d t}=f(P)
$$

Figure 5 shows the temperature response of the 8-wire SMA bundle. It can be seen that the time taken to reach the initial point of transformation decreases as energising current increases. However, by considering the total temperature profile as an approximated first order response, it is shown that the time constant value for the temperature rise, $\tau(T)$, remains constant for changing values of $P$.

$$
\tau_{P 1}(T)=\tau_{P 2}(T) \ldots \ldots=\tau_{P 5}(T)
$$

In the case outlined in Figure $5, P 1>P 2 \ldots>P 5$, so it can be stated that

$$
\frac{d\left(\Delta T_{1}\right)}{d t}>\frac{d\left(\Delta T_{2}\right)}{d t} \ldots \ldots>\frac{d\left(\Delta T_{5}\right)}{d t}
$$

Since the SMA wires are thermally activated, the rate of contraction \& expansion is a function of the rate of heating.

$$
\frac{d x}{d t}=f\left(\frac{d(\Delta T)}{d t}\right)
$$

Therefore it can be stated that for different levels of power, the $90 \%$ heating response time for contraction, $t_{h 90}$ varies. This is outlined in Figure 6 where 
$t_{h 90}(P 1)<t_{h 90}(P 2) \ldots \ldots<t_{h 90}(P 5)$

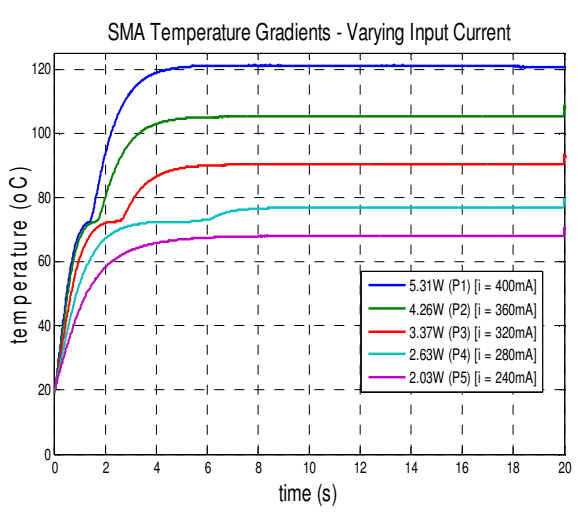

Figure 5: SMA Temperature Gradients

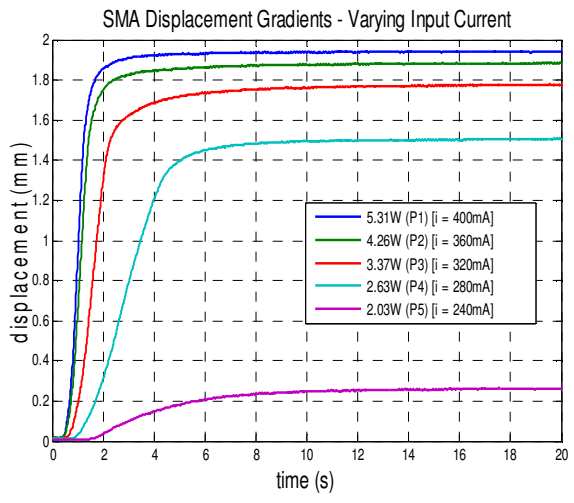

Figure 6: SMA Displacement Gradients

Since the contraction \& expansion of the SMA wires will be used in a novel prosthetic hand design in the generation of grip and release forces via a mechanical framework [6], generated force, F, must also be considered. Analysis of the testbed shows that $\mathrm{F}$ is a time dependent function of $\mathrm{x}$ and it is envisaged that

$$
\frac{d F}{d t}=f\left(\frac{d(\Delta T)}{d t}\right)
$$

Equation (16) demonstrates that the higher the rate of heat transfer to/from the SMA bundle within the prosthetic hand, the faster the contraction time and/or the force generation time.

\section{b) Fixed Current-Varying Bundle Size}

With I remaining constant, from (7) it can be stated that:

$\Delta T \propto R$

It is clear (16) that the greater the number of SMA wires per bundle, the greater the overall resistance to current. This holds true as the wires are connected electrically in series, while they are mounted mechanically in parallel. This results in a faster heating rate (and requires a greater input voltage). Therefore, it can be concluded that the greater the number of parallel SMA wires in a bundle, the faster the contraction time. In order for a prosthetic device to give a consistent output from each individual phalanx of the hand, differing rates of contraction are unsuitable. As a result, $\dot{E}_{\text {in }}$ must be suitably controlled to give consistent and efficient performance. From Ohm's law, it can be seen that by developing a controller that varies the voltage, thereby allowing the current value to remain constant, heating rates can be regulated in SMA bundles across various bundle sizes. 


\section{Forced Cooling of SMA Bundles}

Previous work has shown that the cooling time of the SMA bundles from a strain perspective is significantly larger than that during the heating phase. This suggests that investigation into methods which improve the cooling time of the SMAs is warranted, particularly in prosthetic actuation applications. Forced cooling can be implemented using a variety of techniques including conduction (heat sinking [11]), convection (forced air cooling \& liquid cooling [12]) and radiation (Peltier cooling [13]). The nature of a prosthetic device dictates that many forced cooling techniques are rendered unsuitable for use. A successful cooling system for such a device must be cheap, portable, reusable and safe. These criteria eliminate convection and radiation cooling strategies, leaving conductive cooling in the form of metallic heat sinks, applied at suitable timed intervals, as the most suitable strategy.

Heat sinks were manufactured from three materials, Aluminium $\left(\mathrm{mc}_{\mathrm{p}}=18.3 \mathrm{~J} / \mathrm{K}\right)$, Brass $\left(\mathrm{mc}_{\mathrm{p}}=13 \mathrm{~J} / \mathrm{K}\right)$ and Copper $\left(\mathrm{mc}_{\mathrm{p}}=12.4 \mathrm{~J} / \mathrm{K}\right)$. Each heat sink was individually tested using the cooling module. During the cooling stage, rate of conduction is relatively constant owing to the relatively high heat capacities of the heat sinks. Heat transfer to the sinks follows Fourier's law (17) with convective heat loss being ignored in this analysis.

$\dot{E}_{\text {in }}=-k A\left(\frac{d(\Delta T)}{d x}\right)$
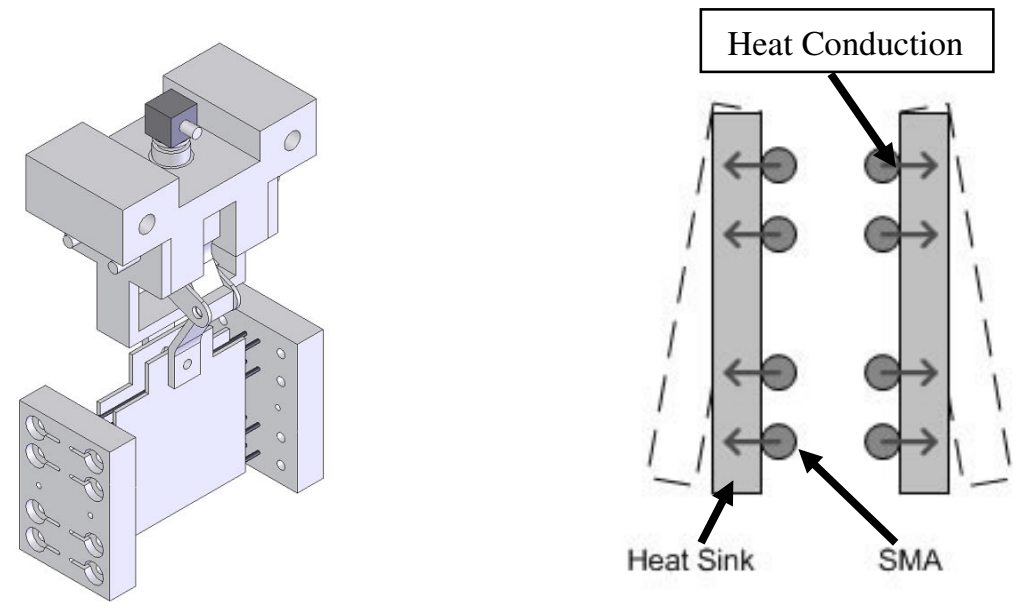

Figure 7 - Cooling Module Attachment (Closed) Figure 8-Heat Conduction using Heat Sink

\section{Experimental Testing}

$\underline{\text { Test } 1}$ - Determination of optimal heat sinking material - Individual tests were completed using a different heat sinking material each time. Testing was carried out in a cyclic manner with the first half 
of the cycle being the heating period. Heat is delivered to the SMA via Joule heating until maximum contraction has occurred. Upon reaching this value, the current is switched off while a heat sink is simultaneously applied to the SMA wires to initiate the cooling phase (Figures $7 \& 8$ ). The heat sink acts to conduct heat away, thus improving the rate of cooling. A test without heat sinks (natural cooling) was also carried out and used as an experimental control.

$\underline{\text { Test } 2}$ - Assessment of chosen heat sink with variable input power - The most suitable heat sink was tested further by heating the SMA bundles using different energising currents. The heat sinks were then applied to cool the bundle. This helps in establishing the cooling times for various SMA steady state temperatures.

$\underline{\text { Test } 3}$ - Analysis of complete cycle time - Cooling of SMAs using the most suitable heat sink was compared to cooling via natural convection. The time to complete a full actuation cycle for the SMA bundle subjected to forced cooling was measured and compared to the complete cycle time of the SMA bundle undergoing natural cooling.

\section{Results:}

The results from Test 1 show that the cooling time is reduced significantly through the use of heat sinks (Figure 9). Aluminium proved to be the most beneficial, reducing the $90 \%$ cooling time, $t_{c 90}$, to approximately 1.9 seconds, compared to 3.4 seconds for natural cooling. Brass exhibited a $t_{c 90}$ value of 2.2 seconds and Copper produced a $t_{c 90}$ value of 2.45 seconds. In addition to Aluminium proving to be the most effective heat sink, it also exhibits additional favourable properties such as low weight and low cost. This makes it suitable for prosthetic applications. Test 2 was carried out using currents up to a maximum of $400 \mathrm{~mA}$, which is rated as $100 \%$ of the recommended current for a $150 \mu \mathrm{m}$ Nitinol wire by the manufacturers [14]. It is demonstrated that the $90 \%$ cooling time has a maximum value of 1.9 seconds with the application of Aluminium heat sinks (assuming excess heat is dissipated from the heat sinks) and decreases as energising currents decrease. The following relationship is observed:

$$
I=157.5\left(t_{c 90}\right)^{2}-9.2\left(t_{c 90}\right)+220
$$




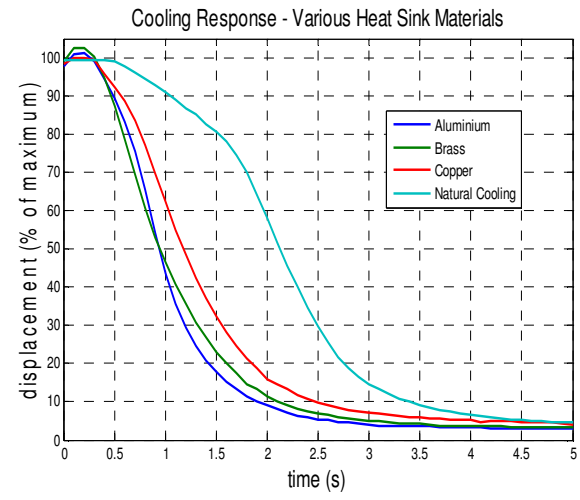

Figure 9: Cooling Response due to Heat Sinks

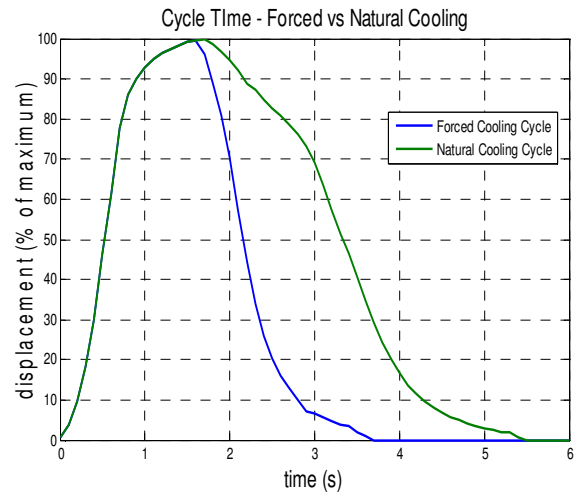

Figure 10: Forced (Al) \& Natural Cooling

Potapov and Da Silva [10] stated that cooling time places limitations on the maximum possible frequency of actuation. In order to increase the frequency of actuation, cooling times must be decreased. Heating rate is a function of energising current and SMA wire resistance, and as such cannot be increased further with fixed actuator parameters. The results of a cycle time analysis are illustrated in Figure 10. The results show that the frequency of actuation can be significantly improved using heat sinks during the cooling phase. The heating phase remains unaffected. It is shown that the use of Aluminium heat sinks permits full actuation cycles at $0.3 \mathrm{~Hz}$, in comparison to natural cooling, where actuation cycles are observed at a maximum rate of $0.18 \mathrm{~Hz}$.

It is predicted that during continuous dynamic cycles, heat is intermittently conducted regularly from the SMA wires to the heat sinking material, and will result in an increase of temperature of the heat sink over time. This will lead to the overall temperature difference $(\Delta T)$ diminishing, decreasing the capacity of the heat sink to conduct heat away from the SMAs. This will be the focus of future work by this group.

\section{Conclusions and Future Work}

An analysis of heat transfer and the relationship between temperature, displacement, force generation and electrical power of single SMAs and SMA bundles has been completed. The conductive forced cooling technique of heat sinking was tested with the SMA bundle in an attempt to improve the dynamic performance of the actuator.

SMAs are thermally activated devices. It is known that a small thermal boundary region encompasses each individual wire. When SMA wires are placed in a parallel bundle formation, the degree of interaction, if any, depends on the closeness of adjacent wires. The results for the 8 wire 
bundle described within this work demonstrate that there is no interaction between the wires as the convective heat transfer coefficient remains relatively unchanged for a single SMA wire and a bundle of SMA wires at steady state conditions. It can therefore be concluded that during the analysis of heat transfer during the transient portion of the heating and cooling cycle, a bundle of parallel SMA wires can be represented as a single SMA wire, with a length equal to the total length of all the parallel SMAs added together. Increasing the number of wires will offset losses in force due to any mechanical inefficiency in the prototype but will result in higher power requirements. Forced cooling via metallic heat sinks of SMAs has been shown to significantly improve the transient response during the cooling (expansion) stage.

Immediate future work will focus on dynamic analysis of the heat sink under long term cyclic loading. The long term focus will be on the development of a suitable controller for the effective control of the SMA bundle in the rig described, using the values \& relationships developed herein.

\section{References}

[1] H. Huang, L. Jiang, D. W. Zhao, J. D. Zhao, H. G. Cai, H. Liu, P. Meusel, B. Willberg, and G. Hirzinger, "The development on a new biomechatronic prosthetic hand based on under-actuated mechanism," Beijing, China, 2006, pp. 3791-3796. L.-R. Lin and H.-P. Huang, "NTU hand: A new design of dexterous hands," Journal of Mechanical Design, Transactions of the ASME, vol. 120, pp. 282-292, 1998.

[3] J. Butterfass, G. Hirzinger, S. Knoch, and H. Liu, "DLR's multisensory articulated hand. ," in IEEE International Conference on Robotics and Automation, 1998, pp. 2081-2086

[4] C. S. Lovchik and M. A. Diftler, "The Robonaut hand: a dexterous robot hand for space," in IEEE International Conference on Robotics and Automation, 1999, pp. 907-912.

J. L. Pons, E. Rocon, R. Ceres, D. Reynaerts, B. Saro, S. Levin, and W. Van Moorleghem, "The MANUS-HAND Dextrous Robotics Upper Limb Prosthesis: Mechanical and Manipulation Aspects," Autonomous Robots, vol. 16, pp. $143-163,2004$.

[6] K. T. O'Toole and M. M. McGrath, "Mechanical Design and Theoretical Analysis of a Four Fingered Prosthetic Hand Incorporating Embedded SMA Bundle Actuators," International Journal of Mathematical, Physical and Engineering Sciences, vol. 1, pp. 83-90, 2008.

[7] K. T. O'Toole, M. M. McGrath, and D. W. Hatchett, "Transient characterisation and analysis of shape memory alloy wire bundles for the actuation of finger joints in prosthesis design " Mechanika, vol. 6, pp. 65-69, 2007.

[8] S. Szykowny and M. H. Elahinia, "Heat transfer analysis of shape memory alloy actuators," Chicago, IL, United States, 2006, p. 8 . 
[9] A. Bhattacharyya, D. C. Lagoudas, Y. Wang, and V. K. Kinra, "On the role of thermoelectric heat transfer in the design of SMA actuators: theoretical modeling and experiment," Smart Materials and Structures, vol. 4, pp. 252-263, 1995.

[10] P. L. Potapov and E. P. D. Silva, "Time Response of Shape Memory Alloy Actuators," Journal of Intelligent Material Systems and Structures, vol. 11, pp. 125-134, 2/1 2000.

[11] R. A. Russell and R. B. Gorbet, "Improving the response of SMA actuators," Nagoya, Jpn, 1995, pp. 2299-2304.

[12] S. A. Mascaro and H. H. Asada, "Wet shape memory alloy actuators for active vasculated robotic flesh," Taipei, Taiwan, 2003, pp. 282-287.

[13] J. Abadie, N. Chaillet, and C. Lexcellent, "An integrated shape memory alloy micro-actuator controlled by thermoelectric effect," Sensors and Actuators A: Physical, vol. 99, pp. 297-303, 2002.

[14] Dynalloy, "Flexinol," in http://www.dynalloy.com. 